\title{
Scaling Version of Kundur's Two-Areas System for Electromechanical Oscillations Representation
}

\author{
Daniel Baltensperger, Jean Dobrowolski, Artjoms Obushevs, Felix Rafael Segundo Sevilla, Petr Korba \\ Zurich University of Applied Science \\ Institute of Energy Systems and Fluid Engineering \\ Winterthur, Switzerland \\ balp(dobr)(obus)(segu)(korb)@ zhaw.ch
}

\begin{abstract}
In this paper, the problem of real implementation of a transmission grid was addressed. In today's modern society, electricity is the most important form of energy for industry and society. A reliable and stable electrical power system is therefore essential. The growing amount of renewable energy sources leads to wheeling electrical power over long distances and to the growth in power electronics-based generation. Both affect the behavior of the electrical power system. The objective of this paper is the development of a scaled version of the Kundur's two areas system in order to implement it in a real laboratory. Additionally, this setup allows to change the rotational inertia and analyze all terminal quantities with phasor measurement units. The control structure of this island grid should reflect the reality as close as possible. All this together enables to study dynamic phenomena with a real small power system. With this laboratory implementation electromechanical swings such as inter-area oscillations after disconnecting a tie-line, changing loads and generation or tripping a synchronous machine can be shown.
\end{abstract}

Index Terms-Cyber-physical energy system, electromechhanical oscillations, transmission grid, grid stability, dynamic behavior, phasor measurement units

\section{INTRODUCTION}

In today's modern society, electricity is the most important form of energy for industry and society. This will be even more accentuated by the new technologies more and more energy consuming (e.g. electrical vehicles) [1] and with the general trend of massive penetration of renewable sources in power systems and the expected decommissioning of nuclear based energy as in Switzerland [2], the future evolution of power grids is a current topic of interest worldwide.

With this context, the general landscape of the distribution and transmission grid is about to drastically change to a more and more decentralized super-grid with small generators scattered everywhere [3].

In Europe, the interest for super-grids is evident as demonstrated with the interconnection of Turkey in 2010 to the already complex network of continental Europe [4]. In addition, there is the potential to grow the interconnection to include areas such as the Nordic region or the UK grid, profiting from the existing HVDC connections and building even more [5]. However, despite the robustness and reliability that extra-large power systems provide, seldom events like those reported by ENTSO-E in December 2016 on continental Europe, trigger large inter-area oscillation causing severe negative effects on the system [6]. Moreover, some recent research [7] proved that this effect could be accentuate by a massive implementation of renewable sources in the European grid.

Whether these so-called inter-area oscillations are undamped, they could eventually lead to a system collapse. This increased our interest to represent these oscillations with a real demonstrator in order to study and control them.

Since inter-areas oscillations is a transmission grid topic, a real demonstrator is a challenging topic due to distances requirements and generator size. Some models such as the New-England IEEE 39 buses [8] or the initial dynamic model of ENTSO-E allow to simulate such oscillations and to control it. In 1994, the smallest representation of a transmission grid was presented [9] through the so-called two-area model. In this paper, this model, presented in section II, has been scaled for a laboratory version to represent inter-area oscillations.

Work structure is following. In section III, the software and hardware solutions to scale the model while keeping representing the electromechanical oscillations are presented. Section IV presents laboratory scaled system behavior during three tests, representing obtained electromechanical oscillations. The final section $\mathrm{V}$ concludes the paper and gives some potential future works.

\section{Two AREAS INITIAL MODEL}

The two-area system can be seen in Fig. 1. The model is defined and introduced in the textbook of Prabha Kundur [9] from pages 813 to 816 . It is now a standard model for analyzing dynamic phenomena in transmission systems. This model represents a $60 \mathrm{~Hz}$ and 11 buses system with four identical synchronous machines. Each of them has a rating of $900 \mathrm{MVA}$ and $20 \mathrm{kV}$ nominal terminal voltage. The two areas are connected with two parallel weak tie-lines [11]. After the step-up transformer, the voltage is $230 \mathrm{kV}$. Lines length are from $10 \mathrm{~km}$ to $220 \mathrm{~km}$.

In simulation, electromechanical oscillation appears following an event in an area (trip of a line or of a generator for example) or between the areas (trip of a line between buses 7, 8 and 9). Fig. 2 shows one example of an electromechanical oscillation after tripping a line of $110 \mathrm{~km}$ between buses 7 and 8. This phenomena is highly linked to the inertia of the synchronous generators. In general inertia is an important 


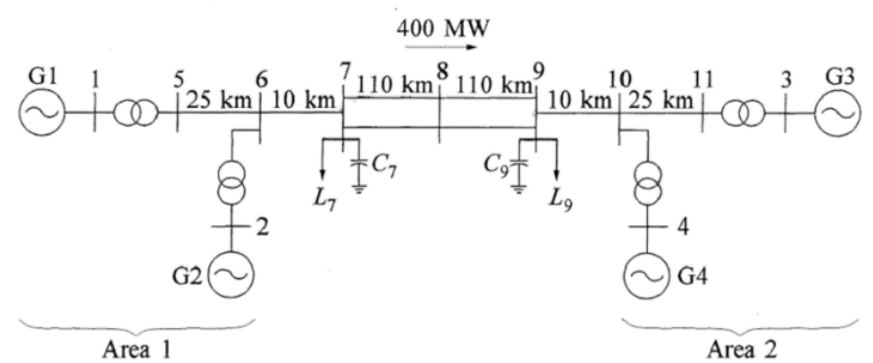

Fig. 1. The original Two-Area System defined by Prabha Kundur [11]

Electromechanical oscillation following a trip of a line

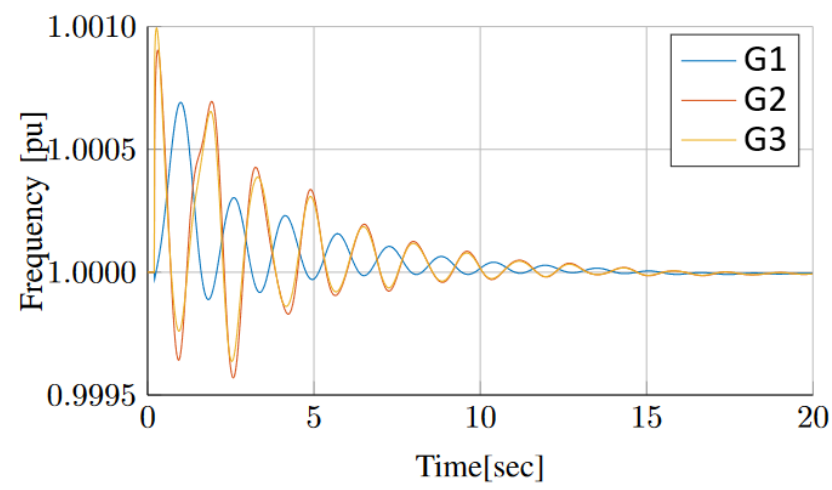

Fig. 2. Electromechanical oscillation following an expected trip of a line in the simulated two-areas model

quantity when studying power system dynamics. This can already be seen with the following two simple examples:

- The widely known swing equation which linked an unbalance between real power generated $\left(P_{m}\right)$ and consumed $\left(P_{e}\right)$, with the dynamic frequency deviation $(\Delta \dot{\omega})$ and the inertia constant $(H)$ :

$$
\Delta \dot{\omega}=\frac{\omega_{0}}{2 H}\left(P_{m}(p . u)-P_{e}(p . u)\right)
$$

With this equation, it is obvious that an increasing inertia constant $\mathrm{H}$ decreases the frequency deviation.

- The second example is the one of small signal rotor angle stability presented similar as in [9] and [10]. Let's consider the single machine infinite bus example depicted in Fig. 3. The real power $P_{e}$ can be written in dependence of the angle $\delta$.

$$
P_{e}=\underbrace{\frac{E^{\prime} E_{B}}{X_{T}}}_{P_{e, \max }} \sin (\delta)
$$

To write the system in state space form we define $x_{1}=\delta$ and $x_{2}=\omega$. For the external inputs we define $u=P_{m}$.

$$
\left(\begin{array}{c}
\dot{x_{1}} \\
\dot{x_{2}}
\end{array}\right)=\left(\begin{array}{c}
x_{2} \\
\frac{\omega_{0}}{2 H}\left(u-P_{e, \max } \sin \left(x_{1}\right)-D x_{2}\right)
\end{array}\right)
$$

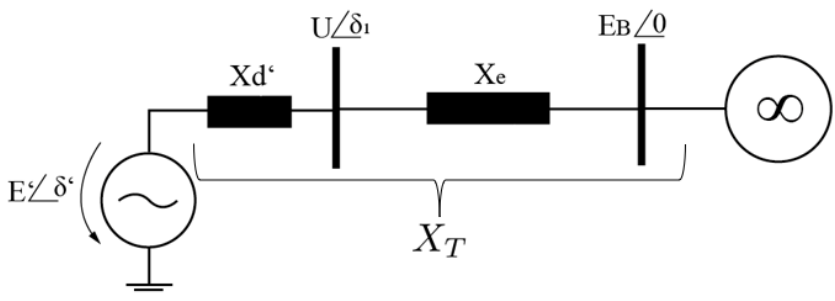

Fig. 3. Single machine infinite bus

To consider the system regarding small signal stability the dynamic system in equation (3) has to be linearized at its equilibrium points $x_{1,0}=\arcsin \left(\frac{P_{m}}{P_{e, \max }}\right)$.

$$
\begin{gathered}
A=\left(\begin{array}{ll}
\frac{\partial f_{1}}{\partial x_{1}} & \frac{\partial f_{1}}{\partial x_{2}} \\
\frac{\partial f_{2}}{\partial x_{1}} & \frac{\partial f_{2}}{\partial x_{2}}
\end{array}\right)=\left(\begin{array}{cc}
0 & 1 \\
-\frac{\omega_{0}}{2 H} P_{e, \max } \cos \left(x_{1,0}\right) & -\frac{D \omega_{0}}{2 H}
\end{array}\right) \\
B=\left(\begin{array}{c}
\frac{\partial f_{1}}{\partial u} \\
\frac{\partial f_{2}}{\partial u}
\end{array}\right)=\left(\begin{array}{c}
0 \\
\frac{\omega_{0}}{2 H}
\end{array}\right) \\
x=\left(\begin{array}{c}
x_{1} \\
x_{2}
\end{array}\right)=\left(\begin{array}{c}
x_{1,0}+\Delta x_{1} \\
x_{2,0}+\Delta x_{2}
\end{array}\right) \Rightarrow \dot{x}=\Delta \dot{x}=\left(\begin{array}{c}
\Delta \dot{x_{1}} \\
\Delta \dot{x_{2}}
\end{array}\right)
\end{gathered}
$$

$$
\Delta \dot{x}=A \Delta x+B \Delta u
$$

In order to make a statement about the dynamic behavior, the eigenvalues of the system have to be considered. To determine the eigenvalues we first calculate the characteristic equation and compare the coefficients with the standard equation for a system second order:

$\lambda^{2}+\frac{D \omega_{0}}{2 H} \lambda+\frac{P_{e, \max } \omega_{0} \cos \left(x_{1,0}\right)}{2 H}=\lambda^{2}+2 \zeta \omega_{n} \lambda+\omega_{n}^{2}=0$

This leads to the following coefficients:

$$
\begin{gathered}
\omega_{n}^{2}=\frac{P_{e, \max } \cos \left(x_{1,0}\right) \omega_{0}}{2 H} \\
\zeta=\frac{D \omega_{0}}{\left.\sqrt{8 H P_{e, \max } \omega_{0} \cos \left(x_{1,0}\right)}\right)}
\end{gathered}
$$

The eigenvalues can finally be written as:

$$
\lambda_{12}=-\frac{D \omega_{0}}{4 H} \pm \sqrt{\left(\frac{D \omega_{0}}{4 H}\right)^{2}-\frac{P_{e, \max } \cos \left(x_{1,0}\right) \omega_{0}}{2 H}}
$$

The following conclusions can now be drawn for this simplified single machine infinite bus example:

- An increasing inertia constant $\mathrm{H}$ decreases $\omega_{n}$ as well as $\zeta$.

- An increasing damping factor D increases the damping ratio $\zeta$. 


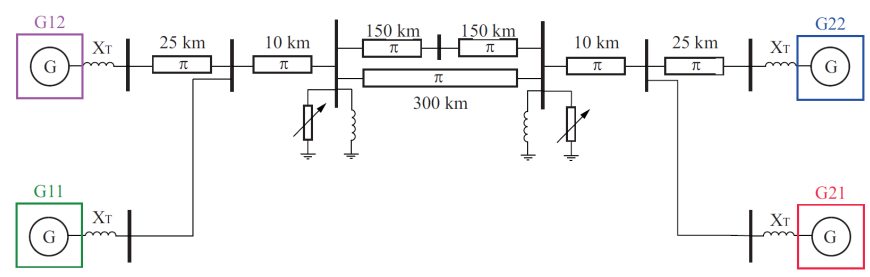

Fig. 4. Single-line diagram of Lab-scale implementation

- An increasing maximum electric power value $P_{e, \max }$ increases the natural frequency and decreases the damping ratio $\zeta$.

With both examples, it is obvious that inertia plays a key role in electromechanical oscillations and has to be represented in the laboratory scaled version.

Analyse of Fig. 1 also immediately shows that another challenge lies in the length of the lines, the size of the generators and the level of voltage for a laboratory scaled version. We will see in section III the solutions found to overcome these problems.

\section{SCALED VERSION (LABORATORY IMPLEMENTATION) AND PHYSICAL SOLUTIONS}

In this section, the scaled version of the two-area model will be presented. The model itself and the differences with the initial Two-areas model are described is sub-section III-A. Then, each subsystems used to physically represent the model in the laboratory are detailed. To finish, the frequency and voltage controls implemented are explained in subsection III$\mathrm{G}$ and III-H.

\section{A. Scaled version vs initial model}

A single-line diagram of the laboratory implementation is presented in Fig. 4 and 5. When comparing Fig. 4 and 1 it can immediately be seen that:

- The transformers are not included.

- Instead of using shunt capacity shunt reactors are used.

In the laboratory the nominal voltage is lower than in the real system. Generator voltage is equal to the lab-scale system voltage. This is why no step-up transformers are included. The transformer of the real system has a certain longitudinal impedance. This is emulated in the laboratory with an additional coil after the generators (XT). To protect the system from high currents, a fuse is installed between generator and coil.

In table I the different properties are listed. All quantities regarding generators and transformers are in p.u. The transmission line quantities are in p.u/km. The base values for the generators in the original system are $S_{\text {Base }}=900 \mathrm{MVA}$ and $U_{\text {Base }}=20 \mathrm{kV}$. Furthermore, the line quantities are based on $S_{\text {Base }}=100 \mathrm{MVA}$ and $U_{\text {Base }}=230 \mathrm{kV}$ [9]. The laboratory base values are $U_{\text {Base }}=400 \mathrm{~V}$ and $S_{\text {Base }}=800 \mathrm{VA}$. Note that the electric frequency in the original system is $60 \mathrm{~Hz}$ and the frequency in the laboratory implementation is $50 \mathrm{~Hz}$.

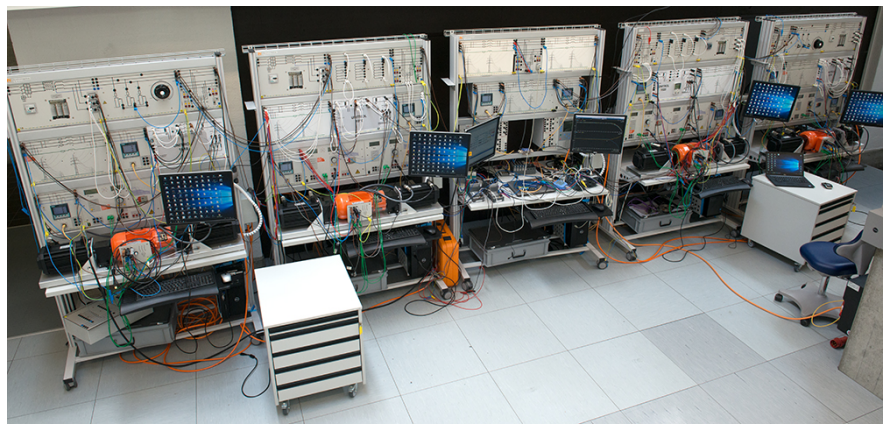

Fig. 5. Lab-scale implementation of Kundur's Two-Area system

TABLE I

LINE AND GENERATOR QUANTITIES IN P.U AND P.U/KM

\begin{tabular}{|c|c|c|c|c|c|c|c|}
\hline \multicolumn{4}{|c|}{ Kundur } & \multicolumn{4}{|c|}{ Laboratory } \\
\hline $\mathrm{Xd}$ & 1.8 & $\mathrm{H} 2$ & 6.175 & $\mathrm{Xd}$ & 1.505 & $\mathrm{H} 2$ & {$\left[\begin{array}{ll}0.22 & 115.76\end{array}\right]$} \\
\hline $\mathrm{Xq}$ & 1.7 & XT & 0.15 & $\mathrm{Xq}$ & 1.355 & XT & 0.2356 \\
\hline $\mathrm{Xd}$ & 0.3 & rline & 0.0001 & $\mathrm{Xd}$ ' & 0.0228 & rline & Table III \\
\hline $\mathrm{Xd} "$ & 0.25 & xline & 0.001 & Xd" & 0.0115 & xline & Table III \\
\hline $\mathrm{Ra}$ & 0.0025 & bline & 0.00175 & $\mathrm{Ra}$ & 0.0515 & bline & Table III \\
\hline H1 & 6.5 & & & H1 & {$\left[\begin{array}{lll}0.22 & 115.76\end{array}\right]$} & & \\
\hline
\end{tabular}

The manufacturer could not provide all model parameters for the machines in the laboratory. The $\mathrm{Xq}$ quantity was estimated with $90 \%$ of the known Xd value [12]. In this paper, it is assumed that the saturation of the original system is the same as of laboratory system. The primary goal of this paper was a real implementation of the Two-areas System. Further quantities are listed in the Kundur books for the original model. They are not listed in this paper because they are still unknown for the here implemented laboratory scaled model. A well-founded parameter identification would have exceeded the scope of this paper.

\section{B. Synchronous machines}

The used machines are from the manufacturer Lucas Nuelle. The different quantities are summarized in table II. These machines are controlled through a servo machine which acts primarily as a motor which takes as an input electrical power from a external grid and transforms it to the output (shaft) as mechanical power. Consequently, it can be said that the motor is used as a turbine emulation.

Another important objective of the servo machine in this project is to emulate a certain level of inertia. As explained in section II inertia has a special importance when analyzing power swings and has to be emulated in this model. To control the servo machine a frequency converter is used which allows controlling the torque or speed via programmable logic controller.

\section{Transmission lines}

In Kundur's Two-Area Model, the transmission lines are highly important. In the laboratory four different lengths of lines are included. Each of them is based on a $\pi$-model structure. For the implementation one $300 \mathrm{~km}$, two $150 \mathrm{~km}$, two $25 \mathrm{~km}$ and two $10 \mathrm{~km}$ transmission lines are used. The long 
TABLE II

SYNCHRONOUS MACHINE PROPERTIES

\begin{tabular}{l|rr} 
Ratings & & \\
\hline Rated voltage (Y/D) & & \\
Rated current & $400 / 230$ & $V$ \\
Rated frequency & $1.5 / 2.6$ & $A$ \\
Rated RPM & 50 & $\mathrm{~Hz}$ \\
Rated power & 1500 & $\mathrm{~min}^{-1}$ \\
Cos( $\varphi$ ) & 0.8 & $\mathrm{~kW}$ \\
Excitation voltage & 1 & \\
Excitation current & 220 & $V$ \\
Inertia & 1.6 & $A$ \\
& 0.0066 & $\mathrm{kgm}^{2}$ \\
Model parameter & & \\
& & \\
Connection & & \\
R1 armature resistance (20 degree) & 10.3 & $\Omega$ \\
Xd synchronous reactance & 301 & $\Omega$ \\
X'd transient reactance & 4.56 & $\Omega$ \\
X"d subtransient reactance & 2.3 & $\Omega$ \\
R2 rotor reactance & 73 & $\Omega$
\end{tabular}

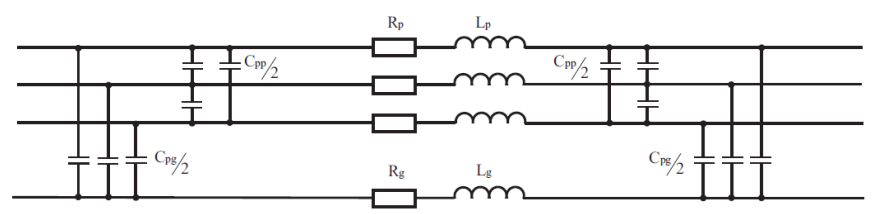

Fig. 6. Line model representation

transmission lines, which connect the two areas, are standard products from Lucas Nuelle. The $10 \mathrm{~km}$ and $25 \mathrm{~km}$ lines were specially manufactured for this project. The line model and the important quantities can be seen in Fig. 6 and table III. Note that the line current in the short lines is strongly limited. This forms a kind of bottleneck in the system.

\section{Circuit breaker}

The circuit breaker is used in the laboratory setup to trip one of the parallel tie-lines, which connects the two areas together. The switch can be open next to the device with a button or remotely by computer. The maximum current is $5 \mathrm{Amp}$ [13]. It is possible to disconnect and connect the different tables in the laboratory. The bus bar and circuit breaker can be seen in figure 7.

\section{E. Loads}

The main load in the laboratory system is a variable ohmic resistance visualized in figure 7 center. It allows changing the resistance between 50 and $750 \mathrm{ohms}$ by hand. Note that the maximal current is resistance dependent and vary between 0.7

TABLE III

LINE MODEL PARAMETER

\begin{tabular}{r|r|r|r|r|r|r|r}
$\mathbf{L}[\mathbf{k m}]$ & $\mathbf{C p p}[\mathbf{n F}]$ & $\mathbf{C p g}[\mu \mathbf{F}]$ & $\mathbf{L p}[\mathbf{m H}]$ & $\mathbf{L g}[\mathbf{m H}]$ & $\mathbf{R p}[\Omega]$ & $\mathbf{R g}[\Omega]$ & $\mathbf{I m}[\mathbf{A}]$ \\
\hline 300 & 600 & 2.2 & 230 & 200 & 7.2 & 15 & 2 \\
150 & 300 & 1.1 & 115 & 100 & 3.6 & 7.5 & 2 \\
25 & 50 & 0.18 & 19 & 17 & 0.6 & 1.2 & 1.2 \\
10 & 8 & 0.03 & 6.25 & 5.7 & 0.2 & 0.4 & 1.2
\end{tabular}
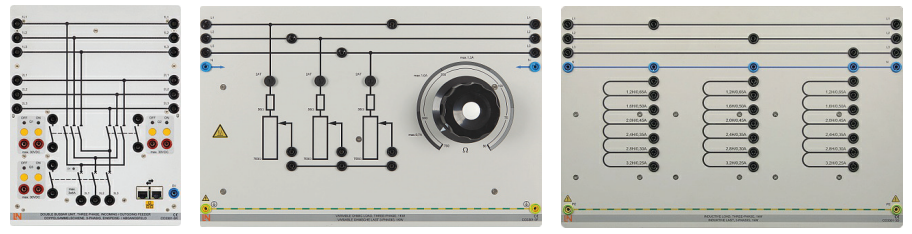

Fig. 7. Left:Circuit breaker; Center:Active load; Right: Inductive load [13]

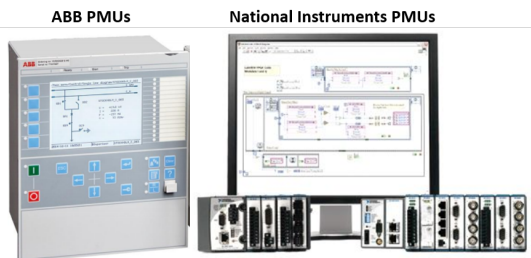

Fig. 8. REE Lab PMUs

- 2 Amp. It is possible to connect the three resistors in delta or Y connection. In this paper, the load is Y connected. The second kind of load is a purely inductive load. It is possible to change the inductivity from $1.2 \mathrm{H}$ until 3.2 in increments of $0.4 \mathrm{H}$. Depending on the adjusted inductivity, the current is limited between 0.65 and $0.25 \mathrm{Amp}$. In this paper, the load is used as a shunt reactor to keep the voltage at the node points in a certain range. This is especially important during the starting phase.

Without shunt reactors the so-caled Ferranti effect appears during the starting phase and the voltage is higher at receiving node than at supply node. If the resistance at receiving node decreases, the current increases and the inductivity in the $\pi$ model starts to consume more reactive power generated by the shunt capacities. Consequently the voltage at receiving node decrease. Two shunt reactors of $3.2 \mathrm{H}$ relax the problem.

\section{F. Measurements}

To identify, analyse and study dynamic phenomena it is essential to receive accurate measurements. In this work Phasor Measurement Units (PMU) from National Instruments and $\mathrm{ABB}$ are used and visualized in figure 8. This device deals with synchrophasor technology. This means that instead of measuring only RMS values of voltage and current the phasor (magnitude plus angle) is measured and transmitted to a control centre every $0.02 \mathrm{~s}$. The phasors are recorded synchronously in different places of the grid and sent to a central control centrum. This allows to make some assessments regarding the state of the power system [15].

In order to analyze electromechanical oscillations each generator is equipped with a PMU to monitor frequency and active power at its terminals. This is visualized in figure 9

\section{G. Frequency control}

The frequency control in the laboratory is done through the PLC functionality of the Lucas Nuelle SCADA software. The frequency controllers are programmed in structured text. The cycle time is not equidistant and between 15 and $20 \mathrm{~ms}$. In 


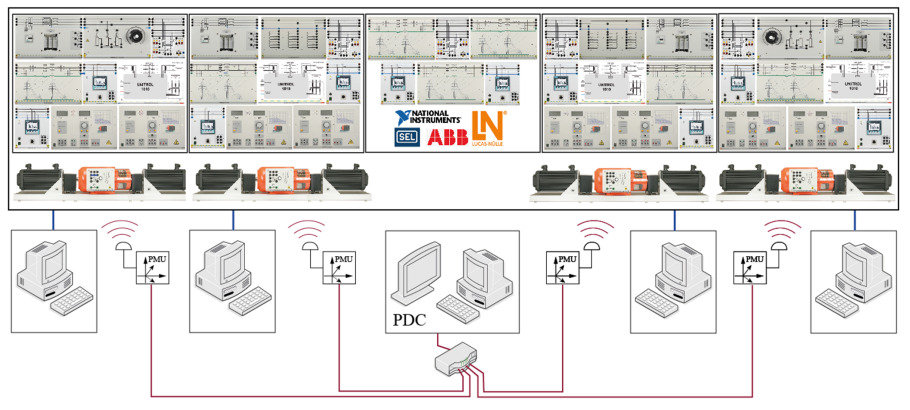

Fig. 9. Kundur's system with communication layer

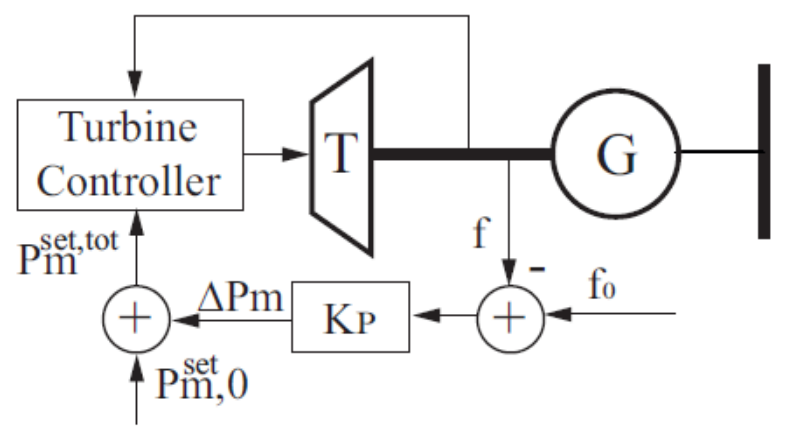

Fig. 10. Technical mimic of the primary control

this paper, we implemented only a primary frequency control as a proportional controller for each machine to represent the so-colled droop control and ensure the sharing of the load between generators [9]. The technical structure is visualised in Fig. 10.

The implementation can be seen as a block diagram in Fig. 11. It is assumed that the user of the laboratory system first brings the system manually to the nominal frequency of 50 Hz. A small deviation from this nominal operation point leads to a control action $\Delta P_{m}$ proportional to the frequency error. The droop value and the frequency deviation together dictate the new torque reference value $T_{r e f}$. The turbine controller given from the manufacturer Lucas Nuelle will increase or decrease the torque until the reference value is reached.

\section{H. Voltage control}

In this implementation each generator is equipped with a static excitation system. To control the voltage, four ABB's Unitrol 1010 [14] devices are included in the laboratory.

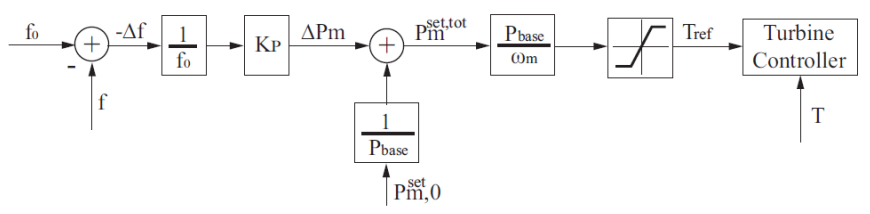

Fig. 11. Block diagram of primary frequency control

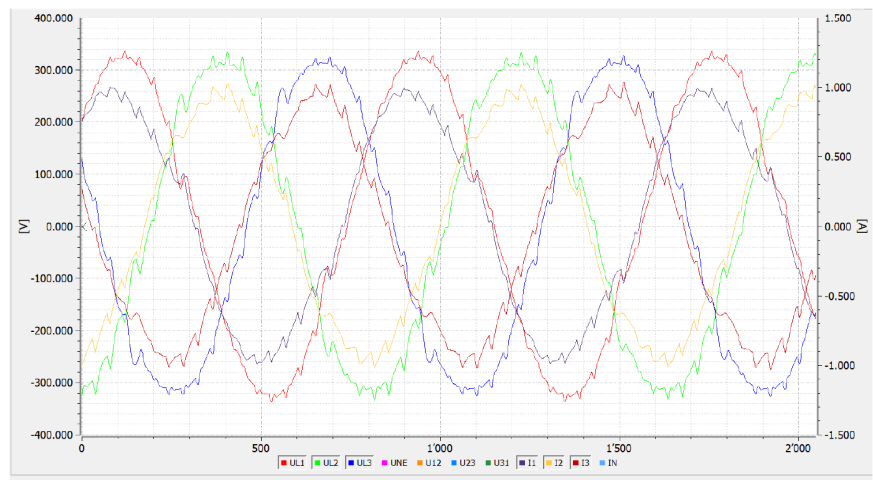

Fig. 12. Terminal currents and voltages

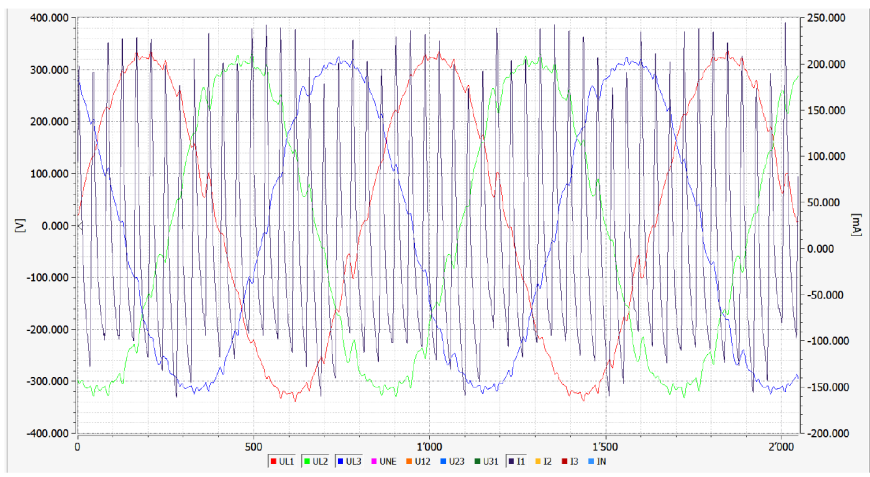

Fig. 13. Excitation current and terminal voltages

The voltage control is done by the AVR functionality of the Unitrol devices. It is a conventional PID controller. Since ABB's Unitrol 1010 are designed for currents up to 10 amperes, connecting these devices to smaller synchronous generators leads to slightly distorted currents and voltages at the measured terminals.

The voltage THD at the terminal is between 4.5 and 6.5 percent and the current THD is in the range of 6 to 9.5 per cent.

In Fig. 12 terminal voltages and currents can be seen. Fig. 13 shows the excitation current and the terminal voltages. It clearly can be seen that the pulse width modulation triggers the harmonics in the terminal voltage.

\section{RESULTS}

In this section, three tests will be presented:

1) System behavior following load event with low inertia

2) System behavior following line event with high inertia

3) System behavior following generator event with high inertia

\section{A. System behavior following load event with low inertia}

The parameter setting for the first scenario is shown in table IV and the active power flow in Fig. 14.

After increasing the load of $\Delta P_{\text {Load }}$ the kinetic energy stored in the rotating masses decreases. This leads to a decreasing frequency and an increasing control action or in 
TABLE IV

PARAMETER SETTINGS PRIMARY FREQUENCY CONTROL

\begin{tabular}{l|rrrr} 
& Gen. 12 & Gen. 11 & Gen. 21 & Gen. 22 \\
\hline Pm0 [W] & 285 & 302 & 270 & 286 \\
Kp & 30 & 30 & 30 & 30 \\
H [s] & 7.84 & 7.84 & 7.84 & 7.84
\end{tabular}

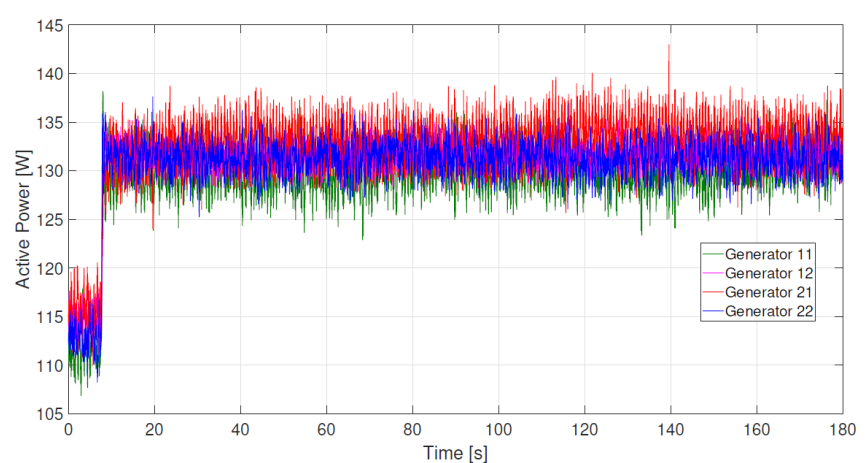

Fig. 14. Active power after a load change with primary frequency control

other words an increasing mechanical power. If the mechanical power is equal the total electrical power the process stops and the frequency becomes steady state. The simple proportional control structure leads to a static error. In this scenario every machine contributes the same. This can be explained by the fact that all machines have the same droop adjusted and furthermore it is assumed that all machines participate in the primary frequency control.

We can clearly see that the signals are noisy. All machines connected to the system in the laboratory are relatively small. Compared with the cycle time of the controller $(20 \mathrm{~ms})$ the machines are fast. This leads to this noisy behavior. Secondly, it can also be seen that there are no electromechanical oscillation observed. This is because the inertia is clearly too low and the generators too small to see this effect.

\section{B. System behavior following line event with high inertia}

When the inertia increases, the electromechanical oscillations can be observed. These kinds of dynamic phenomena appear in laboratory implementation after a disturbance such as a load change, tripping lines or generators. The phenomenon appears based on oscillating voltage angle differences between the generators. Due to the fact that the differences of the voltage angle influence the active power flow directly, these kinds of oscillations can be seen in the active power flow measurements. According to reference [9], it can be distinguished between so-called local oscillations, where oscillations are associated with a single generator or a single plant, and the so-called inter-area oscillations where a group of machines in one part swings against another group of machines in another part of the system. The local modes have usually a frequency between 0.7 and $2.0 \mathrm{~Hz}$ where the frequency of inter-area oscillations is in the range of 0.1 to $0.8 \mathrm{~Hz}$. For the different tests in this subsection and the next one the droop and inertia

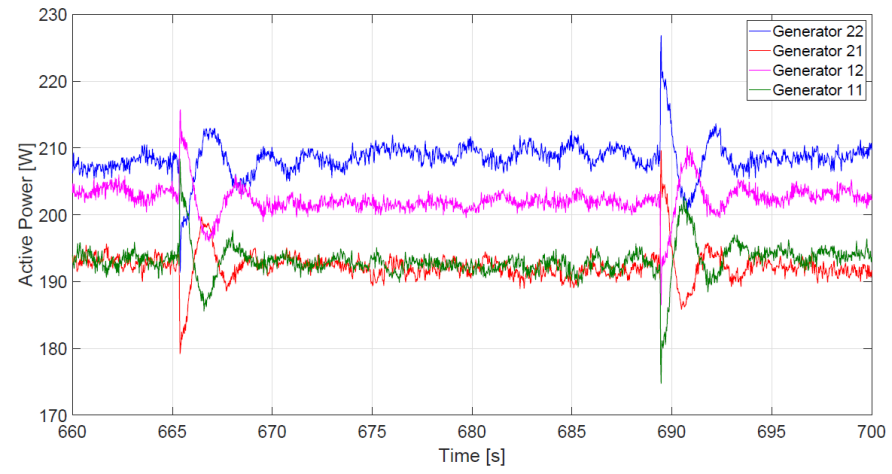

Fig. 15. Active power behavior during disconnection and reconnection of a tie-line

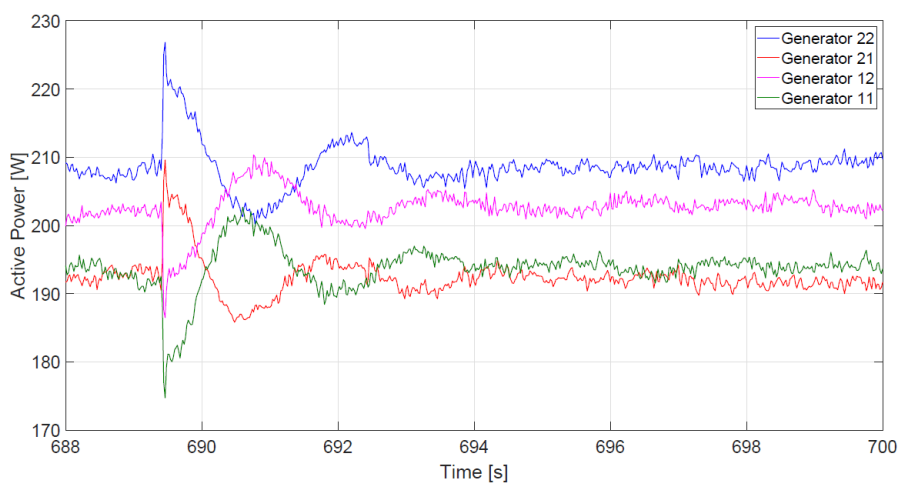

Fig. 16. Active power behavior after reconnecting a tie-line

$(\mathrm{H}=42.25 \mathrm{~s})$ in each machine are equals.

The first analysed disturbance is a trip and reconnection of a tie-line. This can be seen as a sudden change of the tieline impedance. The disturbance is done while 200 watts flow from area two to area one, this is arranged by an uneven split of system load. Fig. 15 shows the active power situation. One tie-line is disconnected after approximately 665 seconds. After 687 seconds the tie-line is reconnected. The power swings can be seen in Fig. 16. We can clearly see that the machines in area one (generator 11 and generator 12) swing again the machines in the area two (generator 21 and generator 22). Due to the fact that two machines in one area swinging against the other two machines in the other area we clearly see here inter-area oscillations. The frequency of the oscillations is approximately $0.37 \mathrm{~Hz}$.

C. System behavior following generator event with high inertia

Another disturbance that can be done in the laboratory is tripping one generator during operation. This was done while 200 watts flowing from area two to area one. In Fig. 17 the power flow situation can be seen. After 721 seconds generator 11 (green) is disconnected. This leads to a loss of generation and consequently a decreasing frequency. The loss of generation is compensated by the other three machines which all participate to primary frequency control. We can 


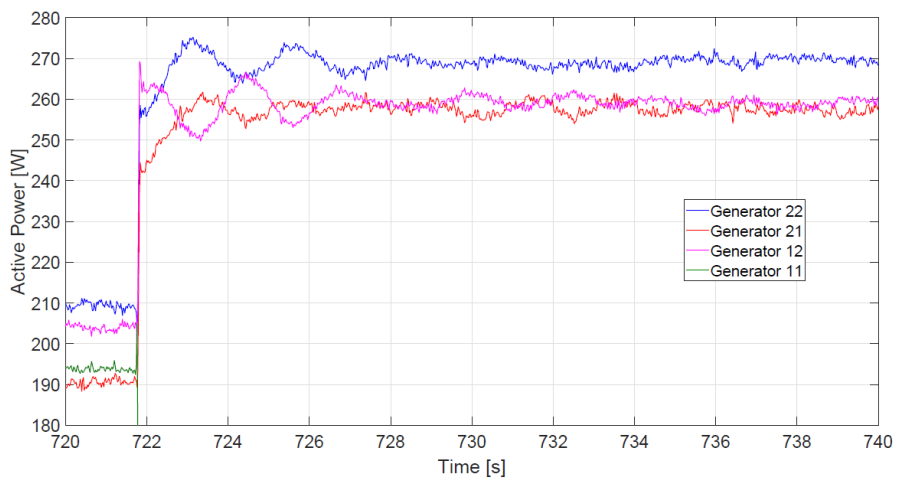

Fig. 17. Active power after tripping a generator

see that after the disturbance the single generator 12 (purple) swings against the two other generators situated in area two.

\section{CONCLUSION AND FUTURE WORKS}

In this paper, we presented a laboratory scaled version of the Kundur's Two-Area System. The objective was achieved and a working small island grid is implemented in the smart grid laboratory in Winterthur. The frequency control is implemented by a programmable logic controller and reflects the reality as close as possible. Furthermore each generator is equipped with a voltage controller from manufacturer $\mathrm{ABB}$ (Unitrol 1010). Most of the system components such as lines, generators, loads and servo machines are from the German manufacturer Lucas Nuelle. Due to the fact that the machines are small further servo machines are integrated to emulate an additional inertia on the rotor of each machine. This allows to study the dynamic behavior of the system for different inertia constants. For measuring and analyzing purposes every machine terminal is equipped with a phasor measurement unit from National Instruments.

Based on this implementation, it is possible to represent electromechanical oscillation in the laboratory but also to study the influence of important quantities such as inertia. Since electromechanical oscillations is an important topic in transmission grid, this demonstrator can be used to test already proposed control such as new PSS of Wide-Area damping controllers [16], [17].

\section{ACKNOWLEDGMENT}

The authors acknowledge the Swiss National Science Foundation (SNSF) under the project number PZENP2 173628 of the program Ambizione Energy Grant (AEG). This research is also part of the activities of SCCER FURIES, which is financially supported by the Swiss Innovation Agency (Innosuisse SCCER program).

\section{REFERENCES}

[1] M.D. Galus and G. Andersson, "Demand Management of Grid Connected Plug-In Hybrid Electric Vehicles (PHEV)," 2008 IEEE Energy 2030 Conference, Atlanta, GA, 2008.

[2] Swiss Federal Office of Energy (SFOE), Energy Strategy 2050, 2017. [Online]. Available: http://www.bfe.admin.ch/energiestrategie2050/index.html?lang=en.
[3] S. Funcke, D. Bauknecht, "Typology of centralised and decentralised visions for electricity infrastructure." Utilities Policy 40 (2016): 67-74.

[4] I.A.Nassar, H. Weber, "System Analysis of the Turkish Power System for Interconnection with Continental Europe",2012.

[5] European Network of Transmission System Operators for Electricity, "10-year network development plan" 2014. [Online]. Available:https://www.rtefrance.com/sites/default/files/141031_tyndp_2014_2.pdf

[6] European Network of Transmission System Operators for Electricity, "Analysis of inter-area oscillations of 1st December 2016", ENTSO-E SG SPD Report, 2017.

[7] [proceeding] C. Rueguer, J. Dobrowolski, P. Korba and F.R. Segundo Sevilla, "Stability Effects After Massive Integration of Renewable Energy Sources on Extra-Large Power Systems", IEEE T\&D PES LA 2020 , Montevideo, Uruguay.

[8] M. Cupelli, C. Doig Cardet, and M. Antonello, "Voltage stability indices comparison on the IEEE-39 bus system using RTDS." In 2012 IEEE International Conference on Power System Technology (POWERCON), pp. 1-6. IEEE, 2012.

[9] P. Kundur, Neal J. Balu, and Mark G. Lauby. "Power system stability and control". Vol. 7. New York: McGraw-hill, 1994.

[10] G. Andersson. "Modelling and Analysi of Electric Power System", ETHZ, 2008

[11] J. Persson. "Kundur's two-area system". STRI, 2004.

[12] Ralf Linnertz. Email conversation from 14.05.2019. "Model parameters for LN synchronous machine".

[13] Lucas Nuelle. "Technical Description of Lucas Nuelle Devices". Lucas Nuelle, 2016.

[14] ABB Excitation Systems. "Unitrol 1020 User Manual. Automatic Voltage Regulator", 2014.

[15] P. Korba, "Elektrische energiesysteme 2". Lecture, 2016.

[16] J. Dobrowolski, F.R. Segundo, and M.R.A. Paternina, "Inter-area Oscillation Control Based on Eigensystem Realization Approach." In 2018 IEEE International Autumn Meeting on Power, Electronics and Computing (ROPEC), pp. 1-6. IEEE, 2018.

[17] J. Dobrowolski, P. Korba, W. Sattinger, and F.R. Segundo Sevilla. "Centralized Wide Area Damping Controller for Power System Oscillation Problems.”, PowerTech Milano, Italy, 2019. 